\title{
Deep Brain Stimulation Frequency of the Subthalamic Nucleus Affects Phonemic and Action Fluency in Parkinson's Disease
}

\author{
Valéria de Carvalho Fagundes, ${ }^{1,2,3}$ Carlos R. M. Rieder, ${ }^{3,4}$ Aline Nunes da Cruz, \\ Bárbara Costa Beber, ${ }^{3}$ and Mirna Wetters Portuguez ${ }^{1,2}$ \\ ${ }^{1}$ Pontifical Catholic University of Rio Grande do Sul (PUCRS), Porto Alegre, RS, Brazil \\ ${ }^{2}$ Brain Institute of Rio Grande do Sul (InsCer), Porto Alegre, RS, Brazil \\ ${ }^{3}$ Hospital de Clínicas de Porto Alegre (HCPA), Porto Alegre, RS, Brazil \\ ${ }^{4}$ Federal University of Health Sciences from Porto Alegre (UFCSPA), Porto Alegre, RS, Brazil \\ ${ }^{5}$ Federal University of Rio Grande do Sul (UFRGS), Porto Alegre, RS, Brazil
}

Correspondence should be addressed to Mirna Wetters Portuguez; mirna@pucrs.br

Received 5 August 2016; Accepted 20 October 2016

Academic Editor: Hélio Teive

Copyright (C) 2016 Valéria de Carvalho Fagundes et al. This is an open access article distributed under the Creative Commons Attribution License, which permits unrestricted use, distribution, and reproduction in any medium, provided the original work is properly cited.

\begin{abstract}
Introduction. Deep brain stimulation of the subthalamic nucleus (STN-DBS) in Parkinson's disease (PD) has been linked to a decline in verbal fluency. The decline can be attributed to surgical effects, but the relative contributions of the stimulation parameters are not well understood. This study aimed to investigate the impact of the frequency of STN-DBS on the performance of verbal fluency tasks in patients with PD. Methods. Twenty individuals with PD who received bilateral STN-DBS were evaluated. Their performances of verbal fluency tasks (semantic, phonemic, action, and unconstrained fluencies) upon receiving low-frequency $(60 \mathrm{~Hz})$ and highfrequency $(130 \mathrm{~Hz})$ STN-DBS were assessed. Results. The performances of phonemic and action fluencies were significantly different between low- and high-frequency STN-DBS. Patients showed a decrease in these verbal fluencies for high-frequency STN-DBS. Conclusion. Low-frequency STN-DBS may be less harmful to the verbal fluency of PD patients.
\end{abstract}

\section{Introduction}

Deep brain stimulation (DBS) at the subthalamic nucleus (STN) improves motor function and the quality of life of patients with advanced Parkinson's disease (PD) [1]. However, some adverse effects are well documented in the literature, such as reduced verbal fluency (VF) [2-9].

The decline in VF observed in PD patients who undergo DBS is not well understood. Studies have hypothesized that this impairment is due to a possible lesion effect from surgery and/or an effect of the neurostimulator parameters, for instance, the frequency of stimulation $[1,2,4,6,8,9]$. Thus, studies assessing the impact of neurostimulation parameters, such as stimulation frequency, on VF are needed.

Low-frequency stimulation has been associated with improved motor symptoms, including freezing of gait and swallowing, in patients with STN-DBS $[10,11]$. Similarly, a study on the effects of low-frequency $(10 \mathrm{~Hz})$ and highfrequency $(130 \mathrm{~Hz})$ DBS-STN on semantic and phonemic verbal fluency found that performance on all VF tasks was significantly better for the low-frequency condition [12]. Further studies are needed to understand how frequency affects VF by analyzing additional VF tasks and the performance of various populations due to language and educational differences.

VF tasks are often used as operating measures of language and executive functions [13]. Among VF tasks are tasks that measure semantic VF (requests for words from a specific semantic group, such as animals or fruits) [13, 14], phonemic VF (requests for words that start with a certain letter) $[13,15]$, verb fluency or action fluency (requests for words designating things that people do) $[13,16]$, and unconstrained VF (requests any word without a criterion) [17]. 
The various VF tasks may provide different types of information regarding cognition because each VF task requires accessing specific lexical and/or semantic representations according to the criteria. The VF tasks activate overlapping areas of the frontal brain regions, but different word retrieval criteria likely activate additional distinct regions [13]. Semantic fluency is thought to be associated with temporal-lobe dysfunction, whereas phonemic fluency is associated with frontal-lobe dysfunction [18]. The action fluency deficit has been reported as a possible marker of frontostriatal impairment $[16,19]$. Although both phonemic and action VF rely on frontal brain areas, evidence has indicated that action VF relies more heavily on semantic information that involves motor content (and because action VF may involve motor brain areas) $[13,19]$, whereas phonemic fluency relies more heavily on lexical retrieval to access words with phonemic similarities [13]. The action fluency task appears to be an important task for evaluating PD, as studies have shown that the action fluency task may be more sensitive to cognitive impairment in $\mathrm{PD}$ patients compared to other VF tasks $[16,19]$. Currently, little is known regarding the neural and cognitive substrates of unconstrained VF because the few studies that have used this VF task only verified the influence of demographic factors on its performance [20, 21]. The absence of a retrieval criterion, as in the unconstrained VF task, may reinforce the need for inhibitory capacity, cognitive flexibility, working memory, and planning. Additionally, the unconstrained VF task may be considered as the absence of specific semantic or lexical involvement.

This study aimed to analyze the impact of low-frequency $(60 \mathrm{~Hz})$ and high-frequency $(130 \mathrm{~Hz})$ STN-DBS on VF tasks of PD patients. We hypothesized that PD patients would present VF deficits due to the frontosubcortical impairment caused by the disease. Thus, if there is an influence of the frequency of stimulation in our population, the frequency should affect the VF tasks that rely more on frontosubcortical functions, as required by the phonemic and action VFs. To test this hypothesis, not only is it important to assess phonemic and action VF tasks, but also the VF tasks that we hypothesized would not be affected by the frequency of stimulation, that is, semantic and unconstrained VFs.

\section{Methods}

2.1. Patients. The present study was a randomized doubleblinded experimental study. The study was conducted with outpatients from the Neurology Service of Hospital de Clínicas de Porto Alegre (HCPA).

The study included 20 patients with idiopathic PD diagnosed according to the criteria of the UK Parkinson's Disease Society Brain Bank [22] and aged between 30 and 75 years. All participants had a bilateral STN-DBS implanted. Only patients with DBS parameters stabilized to the best motor control were included. Also, all patients were Brazilian Portuguese native speakers.

Exclusion criteria included the abuse of illicit drugs or benzodiazepines within the last six months, the presence of auditory impairment, as evaluated by an audiometric screening performed by an audiologist, the presence of visual impairment, a clinical diagnosis of depression or the presence of important signs or symptoms of depression (measured according to the 17-item Hamilton Depression Rating Scale, with a cutoff of 23 for very severe depression) [23], a clinical diagnosis of dementia or Mini-Mental State Examination (MMSE) with scores lower than the expected for the patient's educational level (cutoff of 20 for illiterates, 25 for 1 to 4 years of education, 26.5 for 5 to 8 years, 28 for 9 to 11 years, and 29 for higher levels of education) [24], a history of psychotic symptoms, or a history of alcoholism (screening according to the CAGE questionnaire with a score $\leq 1$ ) [25].

2.2. Procedures. This study was conducted in a randomized, double-blinded manner. The order of the initial DBS conditions was defined by a medical student using the website http://www.random.com/. The order offered by the website was random but with an equal distribution of the initial DBS conditions. The low-frequency $(60 \mathrm{~Hz})$ and highfrequency $(130 \mathrm{~Hz})$ conditions were termed A-condition and B-condition, respectively. Each participant was assigned the $\mathrm{AB}$ order $(n=10)$ or the BA order $(n=10)$. A neurologist adjusted the frequency of stimulation, according to the randomization order, but was not allowed to participate in any rating or evaluation. The participants, the neuropsychologist who administered the VF tasks, and the neurologist who rated the Unified Parkinson's Disease Rating Scale (UPDRSIII) were blinded to the DBS condition. When the experiment was finalized, the A and B codes were revealed to compute the scores in the database.

After adjusting the frequency, the participants waited one hour to carry out the VF tasks. They then performed the following VF tasks: phonemic VF (FAS version and letter $P$ version), semantic VF (animals), unconstrained VF, and action fluency. For the FAS version of the phonemic VF task, the participants were asked to say words beginning with the letters "F," "A," and "S" for one minute for each letter. The final score was the total number of words beginning with " $F$," "A," or "S" that the participants were able to say [12]. For the letter P version of the phonemic VF task, the participants were asked to say as many words as possible beginning with the letter " $P$ " within two minutes $[15,26]$. For the semantic VF task, the participants were asked to say as many animals as possible within one minute [14]. For the unconstrained $\mathrm{VF}$, the participants were asked to say as many words as possible, excluding names and numbers, within 2.5 minutes while keeping their eyes closed [26]. For the action fluency task, the participants were asked to say as many actions or "things that people can do" as possible within one minute $[16,27]$. The instruction of these previous VF did not allow participants to say proper names or numbers. On semantic VF, no score was given for subcategory (e.g., bird) if specific exemplars were also given (e.g., dove, canary). Additionally, sex- and age-specific names of the same animal species were considered to be the same animal (e.g., hen, rooster). On action VF, it was not allowed to use the same verb with different endings (e.g., eat, eating, eaten). Intrusions and perseverations were not scored $[14-16,28]$. The UPDRS-III 
TABLE 1: Baseline characteristics of the participants.

\begin{tabular}{lcc}
\hline Variables & Mean \pm SD or $n(\%)$ & Range \\
\hline Sex, male & $16(80)$ & - \\
Age & $56.65 \pm 10.71$ & $31-75$ \\
Education & $10.10 \pm 5.23$ & $2-22$ \\
Time of disease, years & $15.30 \pm 4.71$ & $10-29$ \\
Levodopa equivalent dose, mg/day & $1165.00 \pm 615.08$ & $300-2300$ \\
Time after surgery, months & $2.21 \pm 1.38$ \\
MMSE & $26.45 \pm 2.52$ \\
Hamilton Depression Rating Scale & & $0-7$ \\
$\quad$ Normal & $14(70)$ & $21-30$ \\
$\quad$ Mild depression & $4(20)$ \\
$\quad$ Moderate depression & $2(10)$ \\
Amplitude $(\mathrm{V})$, left & $3.02(0.65)$ \\
Amplitude $(\mathrm{V})$, right & $2.98(0.60)$ \\
Pulse width $(\mu \mathrm{s})$, left & $79.50(17.61)$ & - \\
Pulse width $(\mu \mathrm{s})$, right & $81.00(17.14)$ & - \\
Frequency $(\mathrm{Hz})$, left and right & $124.00(26.04)$ & $1.80-3.90$ \\
\hline
\end{tabular}

SD: standard deviation; MMSE: Mini-Mental State Examination.

was used for the motor assessment [29]. After performing these evaluations, the neurologist readjusted the frequency of stimulation according to the assigned randomization to evaluate the other conditions. The participants waited one hour to repeat the VF testing and motor assessment. Upon completion of the assessments, the neurologist adjusted the parameters of the STN-DBS implanted back to the stabilized values used by each participant.

Demographic (sex, age, and education), cognitive (MMSE), and clinical (time of disease in years, time after surgery in months, Hamilton Depression Rating Scale, and the levodopa-equivalent dose (LED)) variables were considered in secondary analyses. The LED was measured as $\mathrm{mg} /$ day and was calculated using conversion formulae [30].

The ethics committees of out institution approved this study, and all participants gave written informed consent.

2.3. Statistical Analysis. Statistical analyses were performed using the Statistical Package for Social Sciences (SPSS version 21.0) with a significance level of $5 \%(p \leq 0.05)$. Continuous variables were reported as the mean $(\mathrm{M})$ and standard deviation (SD). Categorical variables were described by the absolute and relative frequencies. The distribution of variables was verified using the Shapiro-Wilk test. To compare the VF performance between $60 \mathrm{~Hz}$ and $130 \mathrm{~Hz}$ frequencies, we used the generalized estimating equation (GEE) model. To verify if any demographic, clinical, or cognitive aspects influenced the effect of stimulation frequency on VF, we conducted Spearman's correlation. We used the delta value of each VF task (VF task score for the $60 \mathrm{~Hz}$ condition minus the VF task score for the $130 \mathrm{~Hz}$ condition) and of the UPDRS-III scores (UPDRS-III score for $60 \mathrm{~Hz}$ minus UPDRS-III score for $130 \mathrm{~Hz}$ ) in the correlational analysis.

\section{Results}

3.1. Patients Characteristics. The initial study sample consisted of 25 individuals; however, 5 were excluded for not meeting the inclusion criteria. Three included patients were not able to complete some of the verbal fluency tasks in both frequency conditions. However, these participants were still included in the data analysis. All participants were on levodopa drugs, only 6 participants were on amantadine (mean dose of $283.33 \mathrm{mg} /$ day), and no one was on anticholinergics or antipsychotics. The baseline characteristics of the participants are presented in Table 1 . Table 2 presents sex, age, the parameters of stimulation, and the VF outcomes of the VF tasks that were significantly different between frequencies of stimulations, for each participant.

3.2. Verification of Practice Effect. The first and second sets of VF tasks were compared to determine if there was a practice/learning effect due to the repetition of the tasks. The results showed that there was no significant difference between the two sets of tasks for any of the VF tasks (Table 3).

3.3. Verification of the Effect of Stimulation Frequency. Table 4 shows that, after $60 \mathrm{~Hz}$ stimulation, the performances of the phonemic (FAS and P version) and action fluency tasks were significantly better than those after $130 \mathrm{~Hz}$ stimulation.

The performances for the VF tasks according to the STN-DBS frequency are shown in Figure 1(a). Despite the significant difference between stimulation conditions for phonemic and action fluencies, the individual performances for each of the VF tasks presented in Figures 1(b), 1(c), and 1(d) indicate that the participants did not exhibit the same outcome pattern. In addition to the comparison analyses, we assessed the distribution of patients who improved or 


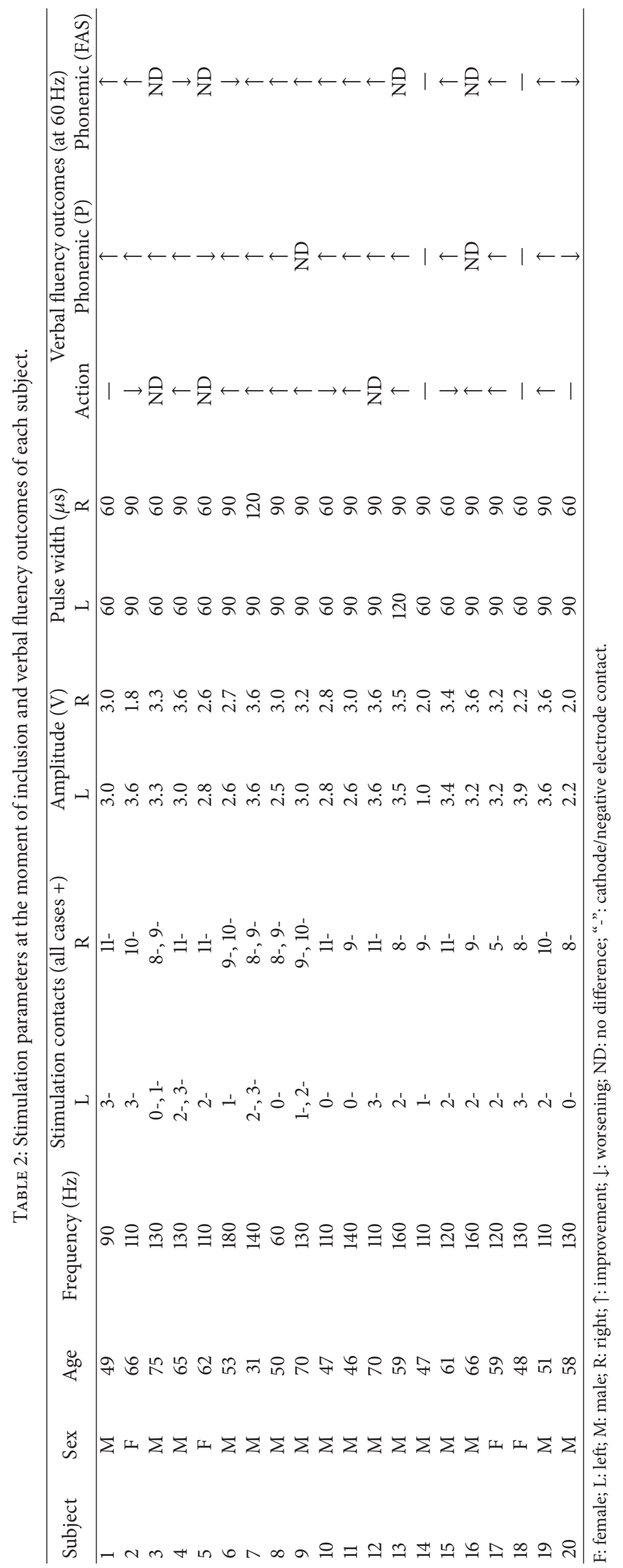


TABLE 3: Comparisons of verbal fluency tasks between moments of administration.

\begin{tabular}{lcccr}
\hline Verbal fluency task & Moment 1 & Moment 2 & Difference & CI 95\% \\
& Mean \pm SD & Mean \pm SD & 0.05 & $-2.96-3.07$ \\
\hline Phonemic, P & $14.53 \pm 1.61$ & $14.47 \pm 1.89$ & -0.11 & $-3.36-3.15$ \\
Phonemic, FAS & $25.26 \pm 2.88$ & $25.39 \pm 15.33$ & -0.16 & $-1.86-1.54$ \\
Semantic, animals & $13.26 \pm 1.07$ & $13.42 \pm 1.22$ & 2.05 & $-1.76-5.87$ \\
Unconstrained & $29.63 \pm 2.50$ & $27.58 \pm 3.00$ & -0.72 & $-2.65-1.21$ \\
Action & $8.39 \pm 1.00$ & $9.11 \pm 1.30$ & 0.856 \\
\hline
\end{tabular}

SD: standard deviation; CI: confidence interval.

TABLE 4: Comparisons of the verbal fluency tasks and UPDRS-III between the different frequencies of SNT-DBS.

\begin{tabular}{lcccc}
\hline Variables & $\begin{array}{c}60 \mathrm{~Hz} \\
\text { Mean } \pm \mathrm{SE}\end{array}$ & $\begin{array}{c}130 \mathrm{~Hz} \\
\text { Mean } \pm \mathrm{SE}\end{array}$ & Difference & CI 95\% \\
\hline UPDRS III, total & $34.33 \pm 4.74$ & $35.44 \pm 4.30$ & -1.11 & $-9.38-7.15$ \\
UPDRS III, tremor & $2.72 \pm 1.20$ & $2.00 \pm 1.09$ & 0.72 & $-2.19-3.63$ \\
UPDRS III, gait & $1.28 \pm 0.26$ & $1.61 \pm 0.33$ & -0.33 & $-0.80-0.13$ \\
UPDRS III, pull test & $1.28 \pm 0.29$ & $1.83 \pm 0.34$ & -0.56 & $-1.12-0.00$ \\
Phonemic VF, P & $16.53 \pm 1.82$ & $12.47 \pm 1.56$ & 4.05 & 0.627 \\
Phonemic VF, FAS & $26.84 \pm 3.36$ & $23.79 \pm 2.79$ & 3.05 & 0.157 \\
Semantic VF, animals & $13.70 \pm 1.20$ & $12.89 \pm 1.08$ & 0.89 & $0.10-6.00$ \\
Unconstrained VF & $29.63 \pm 2.92$ & $27.58 \pm 2.59$ & 1.95 & $-0.76-2.55$ \\
Action VF & $9.94 \pm 1.22$ & $7.56 \pm 1.94$ & 2.39 & $-1.76-5.87$ \\
\hline C: confin & $0.001^{*}$ \\
\hline
\end{tabular}

CI: confidence interval; SE: standard error; UPDRS: Unified Parkinson’s Disease Rating Scale; VF: verbal fluency; ${ }^{*} p \leq 0.05$.

worsened for $60 \mathrm{~Hz}$ stimulation in the phonemic and action fluency tasks. The differences (delta values) in the outcomes of the VF tasks between the frequency conditions $(60 \mathrm{~Hz}$ minus $130 \mathrm{~Hz}$ conditions) were classified as "improvement at $60 \mathrm{~Hz}$ " (positive delta values), "worsening at $60 \mathrm{~Hz}$ " (negative values of delta), or "no difference" (zero values of delta) (Table 5). The individual description of the VF outcomes is also shown in Table 2.

3.4. Correlational Analysis. In the correlational analysis, we included the delta values of the VF task outcomes that showed significant differences between the frequency conditions ( $P$ and FAS versions of the phonemic fluency tasks and the action fluency task) to determine if the difference was correlated with additional variables (including the delta values of UPDRS-III). The delta value of the FAS version of the phonemic VF task was negatively associated with age $(r=$ $-0.0473 ; p=0.041)$. The delta value of the $\mathrm{P}$ version of the phonemic VF task was negatively associated with UPDRS-III $(r=-0.686 ; p=0.002)$ (Table 6$)$.

\section{Discussion}

The present study aimed to investigate the impact of modulating the frequency of STN-DBS on the performance of VF tasks in patients with PD. We assessed the effect of lowfrequency stimulation of $60 \mathrm{~Hz}$ compared to high-frequency stimulation of $130 \mathrm{~Hz}$ in patients who had undergone bilateral STN-DBS in the medication-on state. We found that lowfrequency stimulation had a positive impact on phonemic and action fluency, and this effect was not due to practice. Furthermore, we observed different outcome patterns for the VF tasks based on the frequency conditions, which could not be explained by the demographic, cognitive, and clinical variables that were studied here.

Previous studies have pointed to a decline in VF after STN-DBS surgery in PD patients, although the reason behind this decline is not well understood. There are many methodological differences among such studies, such as evaluations performed while stimulation is "on" or "off," at pre- and postsurgical time points, and with or without a control group [2-9].

Greater declines in VF over time in STN-DBS patients compared to nonsurgical PD patients have been reported, which suggests that VF impairment is related to the DBS intervention [3]. VF may decline as a consequence of microsurgical injuries, which affect the cortical-basal circuits involved in the recovery process of words [31-33]. The number of microelectrode recordings required during surgery for lead placement does not adversely affect VF [4], although this finding does not exclude the possibility of an effect due to the lesions caused by the macroelectrode, suggesting that other factors in addition to microlesions may be involved in VF impairment after surgery.

The frequency of stimulation for treatment of PD has been studied in other clinical situations regarding STNDBS. For example, $60 \mathrm{~Hz}$ stimulation, compared with the routine $130 \mathrm{~Hz}$, improved swallowing function and freezing of gait in patients with PD who underwent bilateral STNDBS $[10,11]$. A previous study that evaluated semantic and 


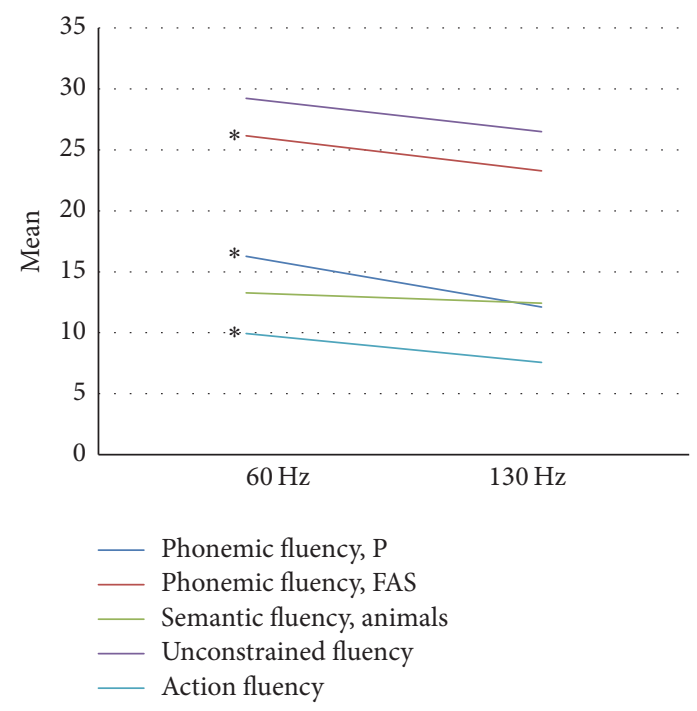

(a)

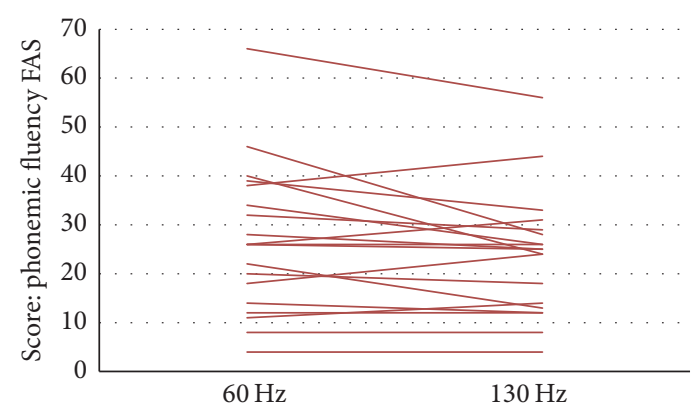

(c)

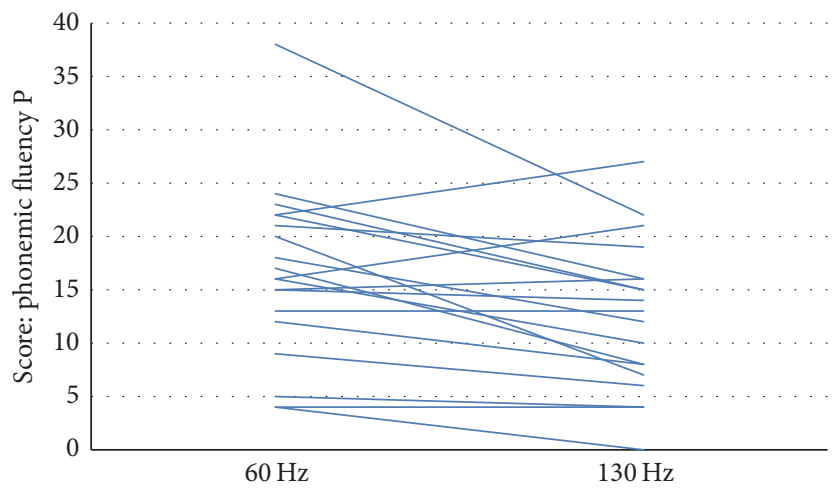

(b)

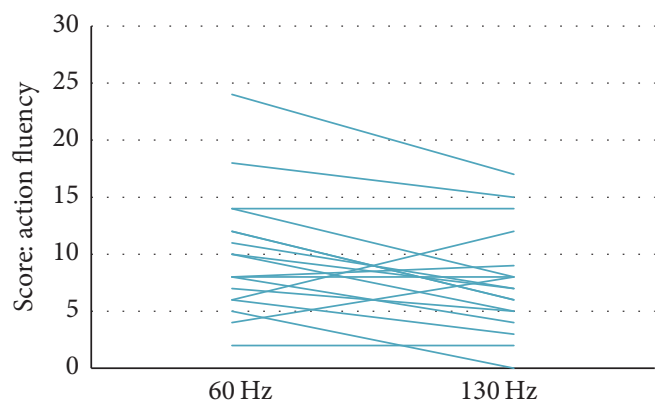

(d)

Figure 1: Patients' performances on VF tasks at low and high stimulation frequencies. (a) Performance of the entire sample for each VF task. * denotes the significant difference between frequency conditions for the respective task. (b) Phonemic fluency performance (P version) by the patient. (c) Phonemic fluency performance (FAS version) by the patient. (d) Action fluency performance by the patient.

TABLE 5: Distribution of delta values for phonemic and action fluency tasks.

\begin{tabular}{lccc}
\hline & $\begin{array}{c}\text { Phonemic VF } \\
\text { P version }\end{array}$ & $\begin{array}{c}\text { Phonemic VF } \\
\text { FAS version }\end{array}$ & Action VF \\
\hline Delta value, mean \pm SD & $4.05(5.50)$ & $3.05(6.73)$ & $2.40(3.60)$ \\
Delta classification, $n(\%)$ & & & $11(55)$ \\
Improvement at $60 \mathrm{~Hz}$ & $14(70)$ & $3(15)$ & $3(50)$ \\
Worsening at $60 \mathrm{~Hz}$ & $2(10)$ & $4(20)$ & $3(15)$ \\
No difference & $2(10)$ & & $3(15)$ \\
\hline
\end{tabular}

SD: standard deviation; VF: verbal fluency.

phonemic VF for $10 \mathrm{~Hz}$ and $130 \mathrm{~Hz}$ stimulation reported greater performances in both VF tasks for low-frequency stimulation [12]. Our results also showed that low-frequency stimulation was associated with better scores of phonemic VF but no improvement was found for semantic VF. Wojtecki et al. [12] did not exclude participants with dementia or lower scores in their cognitive screening, and they did not describe the global cognitive status of the participants. It is known that patients with dementia may also present deficits in semantic VF [16]. Furthermore, differences in language and education may contribute to differences among populations.
When we compared the VF scores and the motor performances between the low-frequency $(60 \mathrm{~Hz})$ and highfrequency $(130 \mathrm{~Hz})$ stimulation trials, we found that phonemic and action fluency significantly declined for $130 \mathrm{~Hz}$ stimulation. However, no significant difference was found between stimulation frequencies for the semantic and unconstrained VF tasks. Our findings indicate that the influence of the stimulation frequency relies more heavily on specific frontosubcortical pathways involved in lexical-word and action-semantic processes, as there was an influence of frequency on phonemic and action VF tasks but not 
TABLE 6: Correlational analysis between VF tasks (phonemic and action), demographic, cognitive, and clinical measures.

\begin{tabular}{|c|c|c|c|c|c|c|}
\hline & \multicolumn{2}{|c|}{ Phonemic - P } & \multicolumn{2}{|c|}{ Phonemic - FAS } & \multicolumn{2}{|c|}{ Action } \\
\hline & $r$ & $p$ & $r$ & $p$ & $r$ & $p$ \\
\hline Age & -0.207 & 0.382 & -0.473 & $0.041^{*}$ & -0.352 & 0.152 \\
\hline Education & -0.192 & 0.416 & 0.300 & 0.212 & -0.021 & 0.935 \\
\hline MMSE & -0.014 & 0.953 & 0.215 & 0.377 & 0.108 & 0.669 \\
\hline Time of disease, years & 0.400 & 0.081 & 0.358 & 0.132 & -0.036 & 0.887 \\
\hline Time after surgery, months & 0.288 & 0.218 & 0.440 & 0.060 & 0.284 & 0.253 \\
\hline Levodopa equivalent dose & 0.351 & 0.140 & 0.131 & 0.592 & -0.003 & 0.992 \\
\hline HDRS, total score & 0.011 & 0.964 & 0.078 & 0.757 & -0.138 & 0.599 \\
\hline UPDRS, total & -0.686 & $0.002^{*}$ & -0.342 & 0.165 & -0.058 & 0.825 \\
\hline UPDRS, tremor & -0.200 & 0.426 & -0.133 & 0.600 & 0.020 & 0.939 \\
\hline UPDRS, gait & -0.378 & 0.122 & 0.004 & 0.986 & 0.003 & 0.990 \\
\hline UPDRS, pull test & -0.312 & 0.207 & -0.479 & 0.068 & -0.321 & 0.209 \\
\hline
\end{tabular}

HDRS: Hamilton Depression Rating Scale; MMSE: Mini-Mental State Examination; UPDRS: Unified Parkinson's Disease Rating Scale; ${ }^{*} p \leq 0.05$.

on semantic and unconstrained VF tasks. As we expected, the frequency affected the VF tasks that rely more heavily on frontosubcortical functions, which are impaired in PD, supporting our a priori hypothesis.

Phonemic and action fluency, which were hampered by high-frequency neurostimulation, are both tasks that involve frontal circuits and that rely more heavily on executive functions. Semantic VF depends on lexical-semantic processes and temporal circuits $[13,34]$. Unconstrained VF is used to assess clinical conditions due to right or left hemisphere lesions, although there are no studies regarding its construct validity and the brain areas involved in adults [17]. One hypothesis regarding the cognitive processing of verbs, based on the theory of embodied cognition, states that the same brain areas involved in planning and motor execution participate in accessing lexical and semantic processes of verbs. This theory, though still controversial, helps to explain the deficits in verb production that have been observed in different clinical groups with Parkinsonian syndromes [35, 36]. STNDBS could affect lexical-semantic processing of actions, such as those involved in the action fluency task; in the same way, it affects neural motor circuitry. The mechanism whereby the stimulus frequency may affect different circuitries remains unknown.

In the present study, no significant difference was found in the motor performance of the patients in relation to the stimulation frequency. One hypothesis for this finding is that the patients were under the effect of dopaminergic medication during the evaluation. If there was no effect of the medication, the low-frequency stimulation $(60 \mathrm{~Hz})$ would be expected to correspond to worse motor symptoms, whereas an improvement in motor performance for the highfrequency stimulation would be expected [10].

The comparison analyses revealed that most patients performed better in phonemic and action VF tasks for the low-frequency condition. However, a few of the participants presented an opposite pattern (worse VF scores for lowfrequency stimulation) or no difference between the conditions. In an attempt to elucidate the cause of these different patterns, correlational analyses were conducted with the delta values of the phonemic and action VF scores. The scores from the FAS version of the phonemic VF task were negatively correlated with age, indicating a possible effect of age on the benefit of low-frequency stimulation; that is, older participants may exhibit lower improvements in performance for low-frequency stimulation compared to younger patients. The scores from the $\mathrm{P}$ version of the phonemic VF task were negatively correlated with UPDRS-III total score, indicating that increased improvements in motor performance were associated with smaller improvements in this phonemic VF task for low-frequency stimulation. This latter finding indicates that motor performance and phonemic VF scores ( $\mathrm{P}$ version) are characterized by opposite outcomes at lowfrequency stimulation. Future studies should seek identifying the factors that explain the different VF improvement profiles for varying frequency stimulation conditions by studying larger samples of PD patients with STN-DBS.

Our study is the first one that called attention to different outcomes of verbal fluency when frequency conditions were compared. Many aspects may influence the modulation of frequency on verbal fluency. Because the STN is thought to have separate functional subregions [37], we hypothesize that the volume and locus of activated STN tissue may interact with the effect of DBS frequency. Besides that, there are studies suggesting that DBS leads to neural plasticity in motor cortex and in modulating corticobasal circuits $[38,39]$. Then, it is possible that frequency of stimulation may also interact with the effect of neural plasticity leading to different outcomes on verbal fluency. These possibilities of interactions may be further investigated in future studies.

Our results should be interpreted in light of some limitations. First, the improvement in the VF task for the lowfrequency condition could be due to an improvement in other cognitive functions, such as attention. However, this study did not evaluate other cognitive functions. We chose to utilize a less-extensive assessment because some patients do not tolerate adjustments in stimulation frequency for long periods of time. Second, the participants were not evaluated in the DBS-off condition so that they were not exposed to unpleasant symptoms for long periods of time, and this study 
did not include a control group. The lack of information for the DBS-off condition and the lack of a control group do not allow surgical effects to be assessed. Third, the administration order of the VF tasks was the same in both conditions. There is the possibility of an order effect, but based on a previous study, we do not believe an order effect occurred, at least pertaining to the action VF task [27].

\section{Conclusion}

In summary, the results of the present study led to two important conclusions. First, the frequency of STN-DBS affects phonemic and action fluency in PD patients. Second, low-frequency $(60 \mathrm{~Hz})$ stimulation is associated with less negative side effects on VF than high-frequency $(130 \mathrm{~Hz})$ stimulation. Therefore, whenever possible, low-frequency stimulation should be the first choice for PD patients, especially for patients who present any cognitive impairments, such as reduced VF, in their daily activities. Future studies utilizing larger sample populations and those incorporating longer study periods should be performed to investigate stimulation effects on VF with regard to electrode position in the STN and other stimulation parameters (amplitude and pulse width).

\section{Competing Interests}

The authors declare that they have no competing interests.

\section{Acknowledgments}

The authors would like to thank the patients for their participation, the Coordenação de Aperfeiçoamento de Pessoal de Nivel Superior (CAPES) for scholarships, and the Fundo de Incentivo à Pesquisa e Eventos (FIPE) from Hospital de Clínicas de Porto Alegre (HCPA) for financial support.

\section{References}

[1] A.-S. Moldovan, S. J. Groiss, S. Elben, M. Südmeyer, A. Schnitzler, and L. Wojtecki, "The treatment of Parkinson's disease with deep brain stimulation: current issues," Neural Regeneration Research, vol. 10, no. 7, pp. 1018-1022, 2015.

[2] K. Witt, O. Granert, C. Daniels et al., "Relation of lead trajectory and electrode position to neuropsychological outcomes of subthalamic neurostimulation in Parkinson's disease: results from a randomized trial," Brain, vol. 136, part 7, pp. 2109-2119, 2013.

[3] D. F. Marshall, A. M. Strutt, A. E. Williams, R. K. Simpson, J. Jankovic, and M. K. York, "Alternating verbal fluency performance following bilateral subthalamic nucleus deep brain stimulation for Parkinson's disease," European Journal of Neurology, vol. 19, no. 12, pp. 1525-1531, 2012.

[4] K. M. Smith, M. O’Connor, E. Papavassiliou, D. Tarsy, and L. C. Shih, "Phonemic verbal fluency decline after subthalamic nucleus deep brain stimulation does not depend on number of microelectrode recordings or lead tip placement," Parkinsonism and Related Disorders, vol. 20, no. 4, pp. 400-404, 2014.
[5] A. Borden, D. Wallon, R. Lefaucheur et al., "Does early verbal fluency decline after STN implantation predict long-term cognitive outcome after STN-DBS in Parkinson's disease?" Journal of the Neurological Sciences, vol. 346, no. 1-2, pp. 299-302, 2014.

[6] F. Ehlen, L. K. Krugel, I. Vonberg, T. Schoenecker, A. A. Kühn, and F. Klostermann, "Intact lexicon running slowly-prolonged response latencies in patients with subthalamic DBS and verbal fluency deficits," PLoS ONE, vol. 8, no. 11, Article ID e79247, 2013.

[7] A. Merola, L. Rizzi, M. Zibetti et al., "Medical therapy and subthalamic deep brain stimulation in advanced Parkinson's disease: a different long-term outcome?" Journal of Neurology, Neurosurgery \& Psychiatry, vol. 85, no. 5, pp. 552-559, 2014.

[8] A. Harati and T. Müller, "Neuropsychological effects of deep brain stimulation for Parkinson's disease," Surgical Neurology International, vol. 4, supplement 6, pp. S443-S447, 2013.

[9] R. Cilia, C. Siri, G. Marotta et al., "Brain networks underlining verbal fluency decline during STN-DBS in Parkinson's disease: an ECD-SPECT study," Parkinsonism and Related Disorders, vol. 13, no. 5, pp. 290-294, 2007.

[10] T. Xie, J. Vigil, E. MacCracken et al., "Low-frequency stimulation of STN-DBS reduces aspiration and freezing of gait in patients with PD," Neurology, vol. 84, no. 4, pp. 415-420, 2015.

[11] T. Xie, U. J. Kang, and P. Warnke, "Effect of stimulation frequency on immediate freezing of gait in newly activated STN DBS in Parkinson's disease," Journal of Neurology, Neurosurgery and Psychiatry, vol. 83, no. 10, pp. 1015-1017, 2012.

[12] L. Wojtecki, L. Timmermann, S. Jörgens et al., "Frequencydependent reciprocal modulation of verbal fluency and motor functions in subthalamic deep brain stimulation," Archives of Neurology, vol. 63, no. 9, pp. 1273-1276, 2006.

[13] S. Pekkala, "Verbal fluency tasks and the neuropsychology of language," in The Handbook of the Neuropsychology of Language, pp. 619-634, Blackwell, Oxford, UK, 2012.

[14] S. M. D. Brucki, S. M. Fleury Malheiros, I. H. Okamoto, and P. H. F. Bertolucci, "Dados normativos para o teste de fluência verbal categoria animais em nosso meio," Arquivos de NeuroPsiquiatria, vol. 55, no. 1, pp. 56-61, 1997.

[15] T. H. Machado, H. C. Fichman, E. L. Santos et al., "Normative data for healthy elderly on the phonemic verbal fluency taskFAS," Dementia e Neuropsychologia, vol. 3, no. 1, pp. 55-60, 2009.

[16] A. L. Piatt, J. A. Fields, A. M. Paolo, W. C. Koller, and A. I. Tröster, "Lexical, semantic, and action verbal fluency in Parkinson's disease with and without dementia," Journal of Clinical and Experimental Neuropsychology, vol. 21, no. 4, pp. 435-443, 1999.

[17] B. Le Blanc and Y. Joanette, "Unconstrained oral naming in left- and right-hemisphere-damaged patients: an analysis for naturalistic semantic strategies," Brain and Language, vol. 55, pp. 42-45, 1996.

[18] S. Schwartz, J. Baldo, R. E. Graves, and P. Brugger, "Pervasive influence of semantics in letter and category fluency: a multidimensional approach," Brain and Language, vol. 87, no. 3, pp. 400-411, 2003.

[19] B. C. Beber and M. L. F. Chaves, "The basis and applications of the action fluency and action naming tasks," Dementia $e$ Neuropsychologia, vol. 8, no. 1, pp. 47-57, 2014.

[20] N. Zimmermann, M. A. de Mattos Pimenta Parente, Y. Joanette, and R. P. Fonseca, "Unconstrained, phonemic and semantic verbal fluency: age and education effects, norms and discrepancies," Psicologia: Reflexao e Critica, vol. 27, no. 1, pp. 55-63, 2014. 
[21] D. M. Jacobsen, Funções executivas na infância: impacto de idade, sexo, tipo de escola, escolaridade parental e sintomas de desatenção/hiperatividade [M.S. thesis], Programa de PósGraduação em Psicologia da Pontifícia Universidade Católica do Rio Grande do Sul, Porto Alegre, Brazil, 2016.

[22] A. J. Hughes, S. E. Daniel, L. Kilford, and A. J. Lees, "Accuracy of clinical diagnosis of idiopathic Parkinson's disease: a clinicopathological study of 100 cases," Journal of Neurology, Neurosurgery \& Psychiatry, vol. 55, no. 3, pp. 181-184, 1992.

[23] C. L. Hooper and D. Bakish, "An examination of the sensitivity of the six-item Hamilton Rating Scale for Depression in a sample of patients suffering from major depressive disorder," Journal of Psychiatry and Neuroscience, vol. 25, no. 2, pp. 178184, 2000.

[24] S. M. D. Brucki, R. Nitrin, P. Caramelli, P. H. F. Bertolucci, and I. H. Okamoto, "Suggestions for utilization of the mini-mental state examination in Brazil," Arquivos de Neuro-Psiquiatria, vol. 61, no. 3, pp. 777-781, 2003.

[25] R. A. do Amaral and A. Malbergiera, "Evaluation of a screening test for alcohol-related problems (CAGE) among employees of the Campus of the University of São Paulo," Revista Brasileira de Psiquiatria, vol. 26, no. 3, pp. 156-163, 2004.

[26] R. P. Fonseca, M. A. D. M. P. Parente, H. Cote, B. Ska, and Y. Joanette, Bateria MAC-Bateria Montreal de Avaliação da Comunicação, Pró-Fono, 2008.

[27] B. C. Beber and M. L. F. Chaves, "Does previous presentation of verbal fluency tasks affect verb fluency performance?" Dementia \& Neuropsychologia, vol. 10, no. 1, pp. 31-36, 2016.

[28] S. M. D. Brucki and M. S. G. Rocha, "Category fluency test: effects of age, gender and education on total scores, clustering and switching in Brazilian Portuguese-speaking subjects," Brazilian Journal of Medical and Biological Research, vol. 37, no. 12, pp. 1771-1777, 2004.

[29] S. Fahn, C. Marsden, D. Calne, and M. Goldstein, "Fahn S, Elton RL. and members of the UPDRS Development Committee. Unified Parkinson's disease rating scale," in Recent Developments in Parkinson's Disease, pp. 153-163, Macmillan Healthcare Information, Florham Park, NJ, USA, 1987.

[30] C. L. Tomlinson, R. Stowe, S. Patel, C. Rick, R. Gray, and C. E. Clarke, "Systematic review of levodopa dose equivalency reporting in Parkinson's disease," Movement Disorders, vol. 25, no. 15 , pp. 2649-2653, 2010.

[31] A. I. Troster, "Neuropsychology of deep brain stimulation in neurology and psychiatry," Frontiers in Bioscience, vol. 14, no. 5, pp. 1857-1879, 2009.

[32] A. I. Tröster, S. P. Woods, and J. A. Fields, "Verbal fluency declines after pallidotomy: an interaction between task and lesion laterality," Applied Neuropsychology, vol. 10, no. 2, pp. 6975, 2003.

[33] A. I. Tröster, J. A. Fields, J. A. Testa et al., "Cortical and subcortical influences on clustering and switching in the performance of verbal fluency tasks," Neuropsychologia, vol. 36, no. 4, pp. 295304, 1998.

[34] M. Pihlajamäki, H. Tanila, T. Hänninen et al., "Verbal fluency activates the left medial temporal lobe: a functional magnetic resonance imaging study," Annals of Neurology, vol. 47, no. 4, pp. 470-476, 2000.

[35] G. Vigliocco, D. P. Vinson, J. Druks, H. Barber, and S. F. Cappa, "Nouns and verbs in the brain: a review of behavioural, electrophysiological, neuropsychological and imaging studies," Neuroscience and Biobehavioral Reviews, vol. 35, no. 3, pp. 407426, 2011.
[36] T. H. Bak, "The neuroscience of action semantics in neurodegenerative brain diseases," Current Opinion in Neurology, vol. 26, no. 6, pp. 671-677, 2013.

[37] A. Mikos, D. Bowers, A. M. Noecker et al., "Patient-specific analysis of the relationship between the volume of tissue activated during DBS and verbal fluency," NeuroImage, vol. 54, supplement 1, pp. S238-S246, 2011.

[38] S. J. Kim, K. Udupa, Z. Ni et al., "Effects of subthalamic nucleus stimulation on motor cortex plasticity in Parkinson disease," Neurology, vol. 85, no. 5, pp. 425-432, 2015.

[39] X.-H. Li, J.-Y. Wang, G. Gao, J.-Y. Chang, D. J. Woodward, and F. Luo, "High-frequency stimulation of the subthalamic nucleus restores neural and behavioral functions during reaction time task in a rat model of Parkinson's disease," Journal of Neuroscience Research, vol. 88, no. 7, pp. 1510-1521, 2010. 


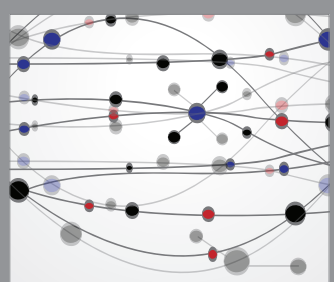

The Scientific World Journal
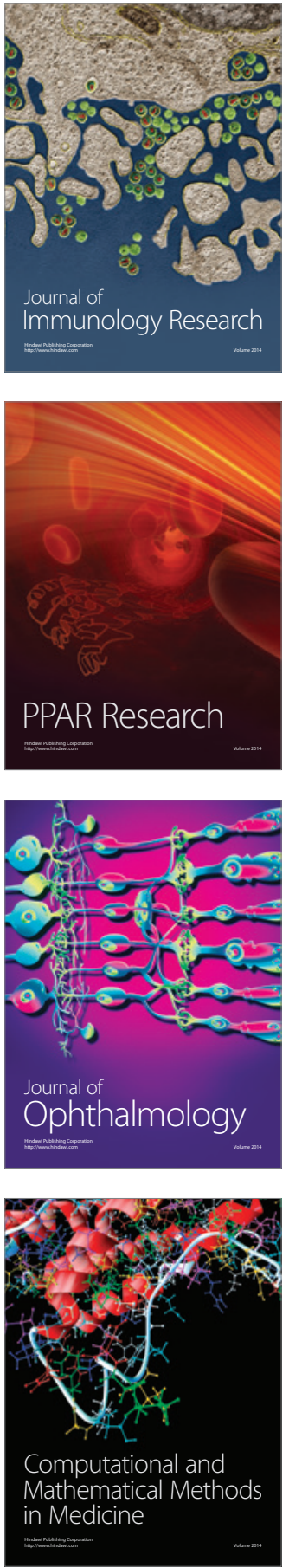

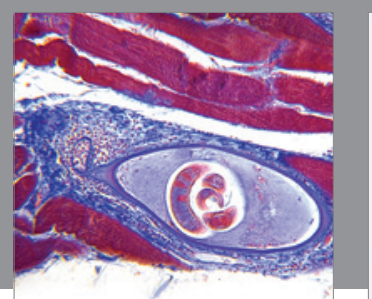

Gastroenterology Research and Practice

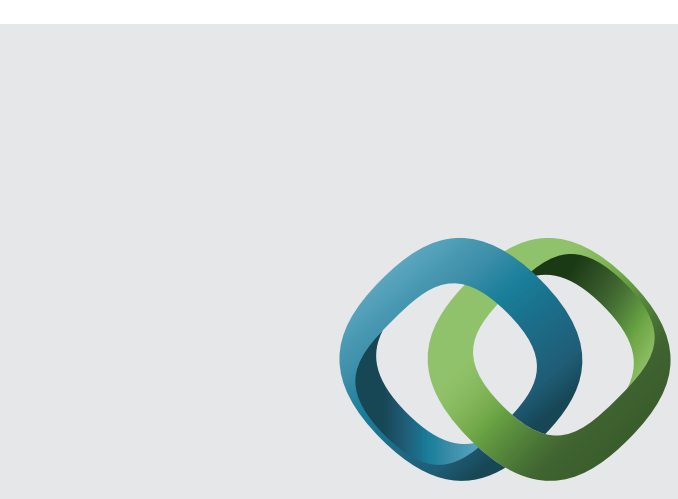

\section{Hindawi}

Submit your manuscripts at

http://www.hindawi.com
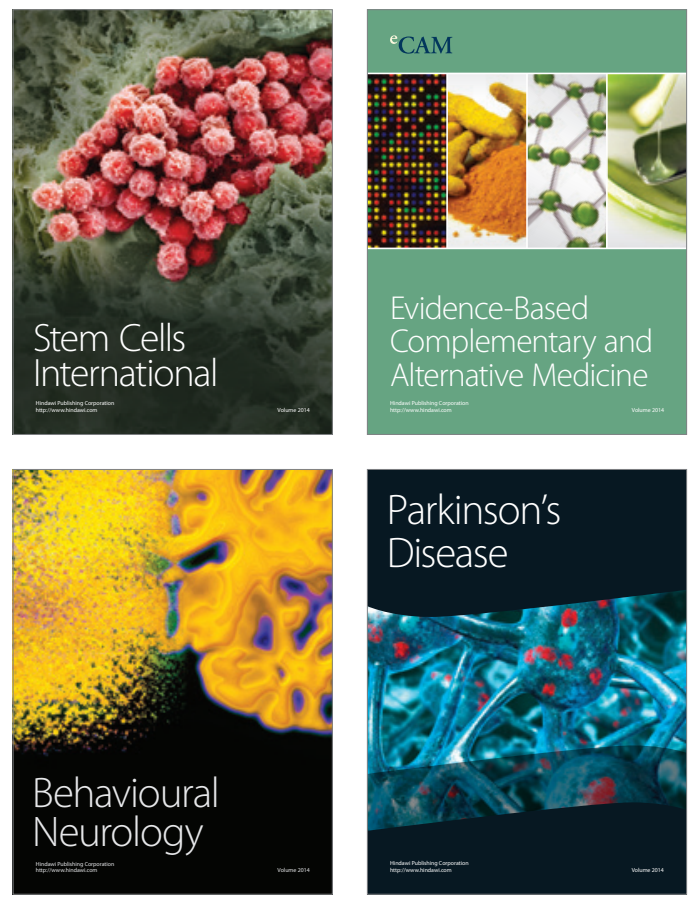
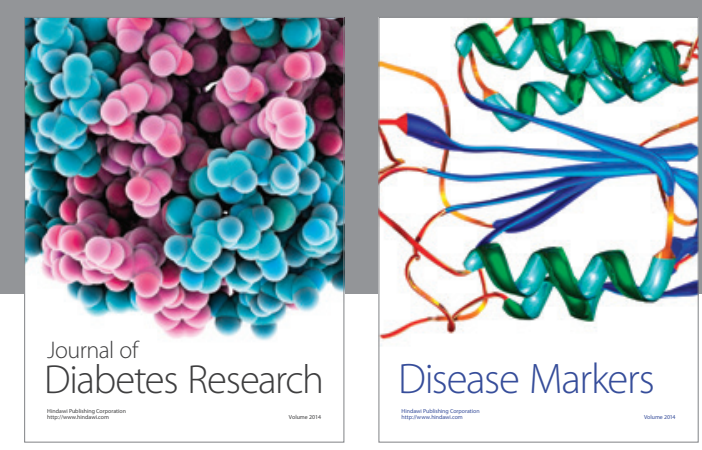

Disease Markers
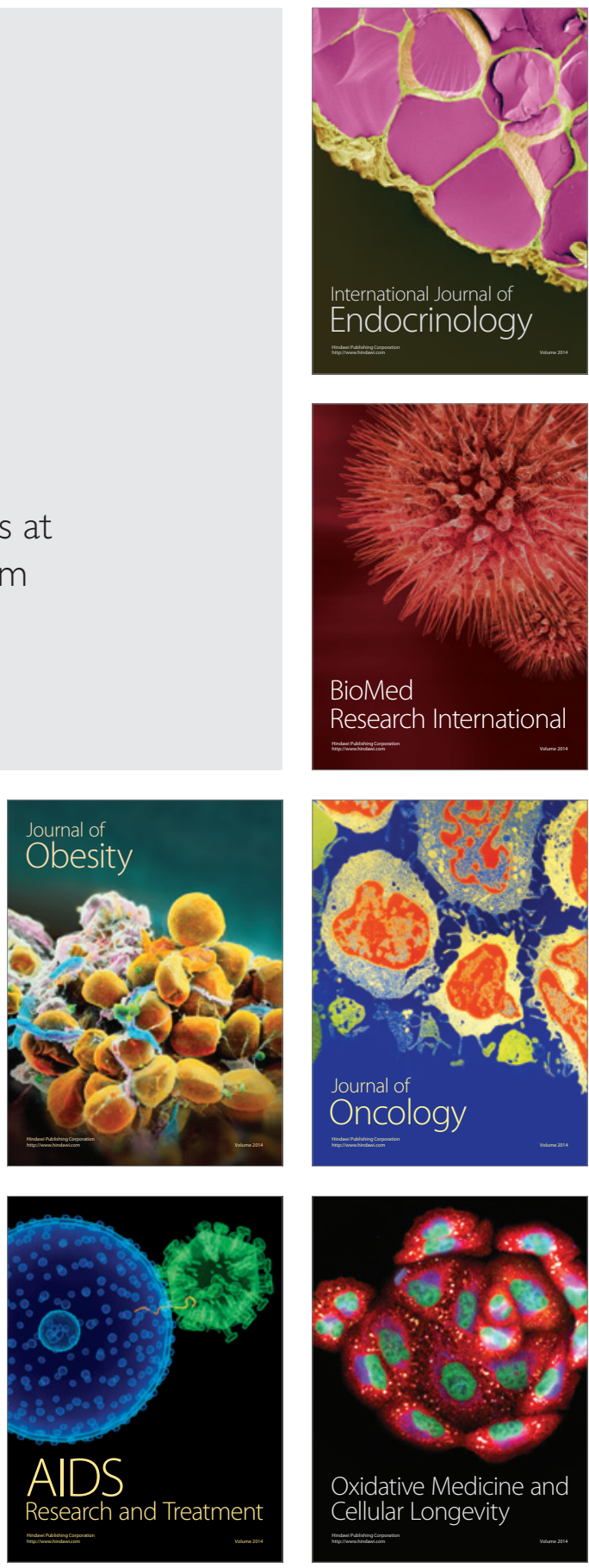